\section{Robotic Harvest of a Right Gastroepiploic Lymph Node Flap}

Pedro Ciudad ${ }^{1}$, Shivprasad Date ${ }^{1}$, Ming-Hsien Lee ${ }^{2}$, Federico Lo Torto ${ }^{1}$, Fabio Nicoli ${ }^{1}$, Jun Araki ${ }^{1}$, Hung-Chi Chen ${ }^{1}$

Departments of ${ }^{1}$ Plastic and Reconstructive Surgery and ${ }^{2}$ General Surgery, China Medical University Hospital, Taichung, Taiwan

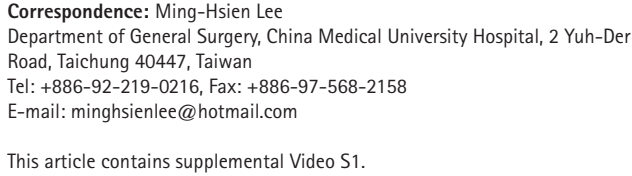

All authors hereby declare no potential conflict of interest and that they did not receive funding for this work from any of the following organizations: National Institutes of Health (NIH), Wellcome Trust, Howard Hughes Medical Institute (HHMI), and other(s).

No potential conflict of interest relevant to this article was reported.

Received: 1 Dec 2015 • Revised: 20 Jan 2016 • Accepted: 7 Feb 2016 pISSN: 2234-6163 • elSSN: 2234-6171

http://dx.doi.org/10.5999/aps.2016.43.2.210 Arch Plast Surg 2016;43:210-212

Copyright (C) 2016 The Korean Society of Plastic and Reconstructive Surgeon This is an Open Access article distributed under the terms of the Creative Commons Attribution Non-Commercial License (http://creativecommons.org/licenses/by-nc/4.0/) which permits unrestricted non-commercial use, distribution, and reproduction in any medium, provided the original work is properly cited.

Lymph node flap (LNF) transfer has proven to be an effective option in the treatment of lymphedema. As a result, much research is presently focused on finding an ideal donor site that results in good clinical outcomes while avoiding iatrogenic lymphedema of the donor area.

The omental flap with its lymph nodes based on the right gastroepiploic vessels that has been described for the treatment of lymphedema in the extremities avoids donor site lymphedema of the extremities. Harvesting this flap laparoscopically further reduces the donor site morbidity $[1,2]$.

Robotic surgery has gained popularity in numerous surgical specialties, including plastic surgery $[3,4]$, due to its advantages over laparoscopic and endoscopic techniques. Herein, we report a novel application of robotic surgery for the harvest of a right gastroepiploic lymph node flap (RGE-LNF) for the treatment of lymphedema of the extremities. To the best of our knowledge, the application of a robotic technique for the harvest of this flap has not been reported in the literature.

A 55-year-old woman presenting with left lower extremity lymphedema and unsatisfactory outcomes following conservative treatment was offered surgical management with RGE-LNF transfer. All aspects of the various options for flap harvest were explained to her, including the open, laparoscopic, and robot-assisted techniques. The patient was comfortable with the robotic option and gave consent for the Da Vinci Surgical System (Intuitive Surgical Inc., Sunnyvale, CA, USA) to be used for flap harvest (Supplemental Video S1, which demonstrates the robotic RGE- LNF harvest).

An experienced general surgeon trained in robotic surgery performed the harvest of the flap with guidance from the plastic surgery team. One $12-\mathrm{mm}$ supraumbilical port was created with an optical trocar for camera placement. After abdomen insufflation, two 8 -mm ports were inserted at least $8 \mathrm{~cm}$ laterally from and $5 \mathrm{~cm}$ below the supraumbilical port, one on the right and the other over the left lower abdomen. Finally, a 5-mm assistance port was inserted in the epigastric region slightly cephalad to the camera port and midway between the camera and the right-side port. The RGE-LNF is based on the right gastroepiploic artery and vein (Fig. 1). Exploration was started by identifying the omental attachment to the greater curvature of the stomach and transverse colon, followed by identification of the right gastroepiploic vessels. Flap dissection was initiated by detaching the omentum from the transverse colon. The vertical gastric branches at the cranial aspect of

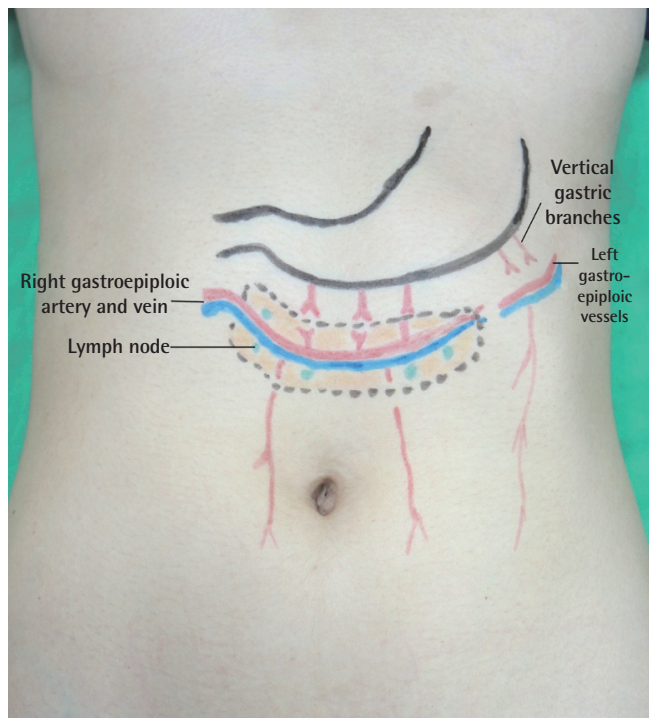

Fig. 1.

Anatomical landmarks of the right gastroepiploic lymph node flap. The flap is based on the right gastroepiploic vessels and includes the omental tissue and embedded lymph nodes between the gastric and colonic attachment of the omentum. 


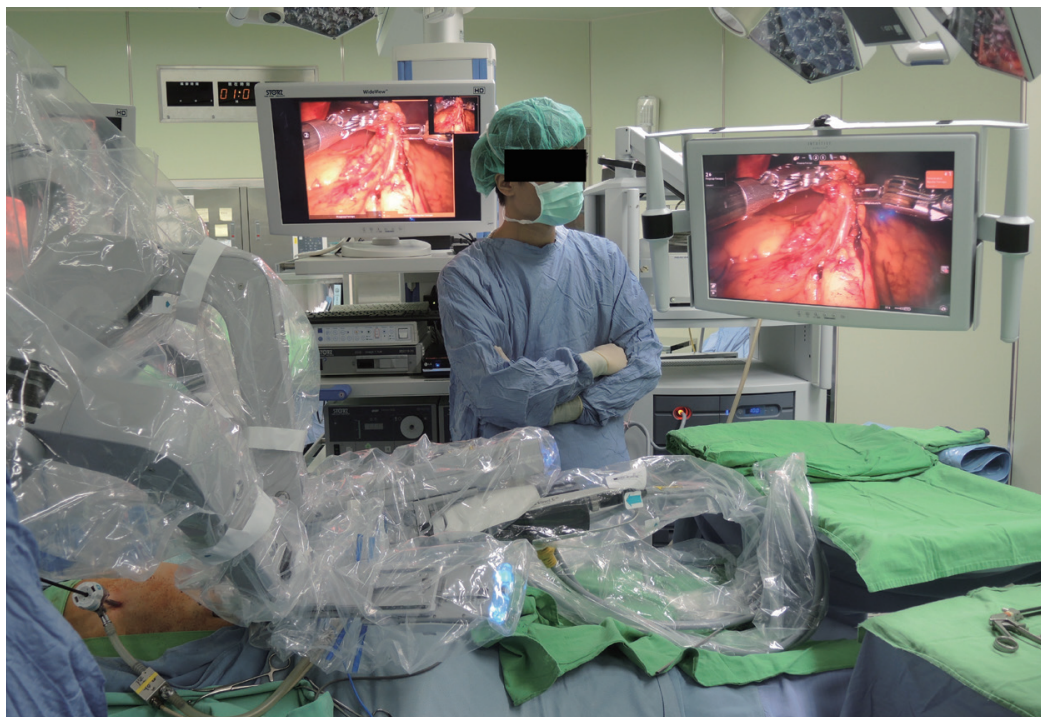

Fig. 2.

Photograph of the surgical procedure. The robot-assisted apparatus provided three-dimensional visualization, improving the precision and the lowering risk of vascular pedicle injury.

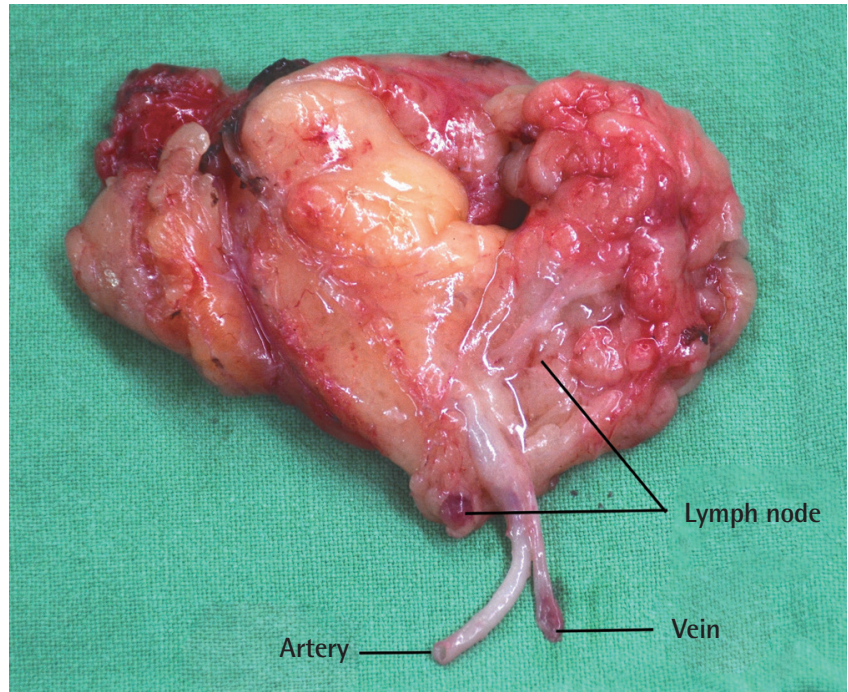

Fig. 3.

The right gastroepiploic lymph node flap on a side table prior to the commencement of microanastomosis. The lymph nodes are clearly visible within the flap tissue.

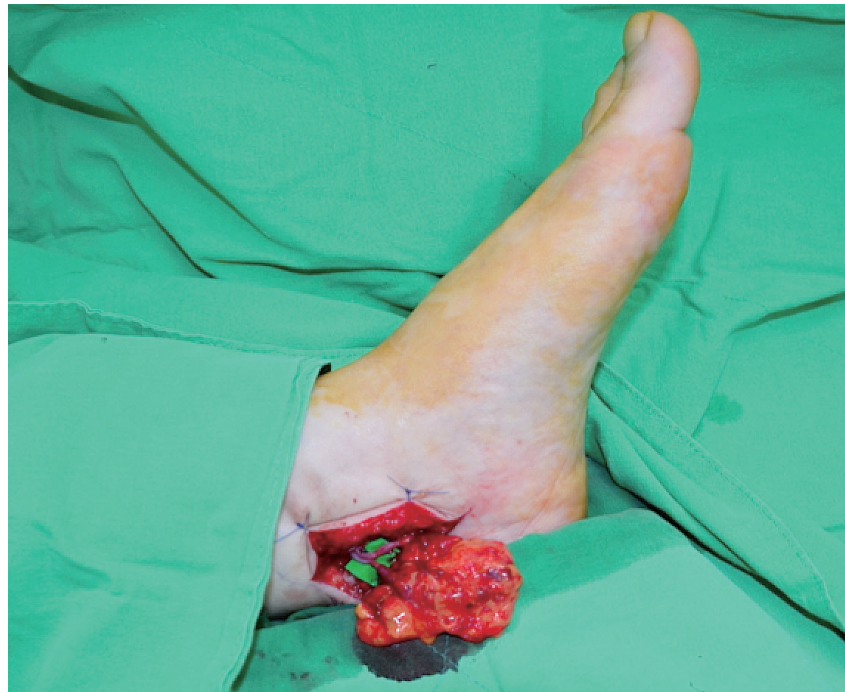

Fig. 4.

Inset of the right gastroepiploic lymph node flap at the ankle level and microanastomosis with the medial plantar recepient vessels. the flap were serially ligated while maintaining a flap width of approximately $5 \mathrm{~cm}$. The craniocaudal dissection was completed, keeping the flap centered on the vascular pedicle. Dissection then proceeded to the left subcostal region where the left gastroepiploic vessels were ligated. Special attention was paid to include the lymph nodes, lymphatics, and the surrounding omental tissue during the flap harvest (Fig. 2). A flap with a length of $10 \mathrm{~cm}$ was dissected up to the origin of the right gastroepiploic vessels, remaining parallel to the greater curvature of the stomach. Finally, the right gastroepiploic artery and vein were carefully dissected, isolated, and ligated.

The total time of flap harvest was 55 minutes. The flap was then exteriorized and transferred to the lymphedematous recipient site (Fig. 3). Under the operating microscope, the flap was minimally trimmed at the margins, avoiding any damage to the lymphatic tissue witin the flap. Microsurgical anastomoses were performed at the ankle level using the medial plantar vessels in an end-to-end fashion after adequate vessel preparation (Fig. 4). The procedure was uneventful and the patient remained comfortable during the perioperative period.

The use of robotic surgery technologies, such as the Da Vinci system, has increased in various fields, and the distinct advantages of robotic surgery have been widely reported in the literature [4]. Likewise, when 
comparing the robot-assisted technique with the laparoscopic approach for the RGE-LNF harvest, we noted that the robot-assisted intraperitoneal approach had specific advantages. The three-dimensional optics of the Da Vinci system allowed superior visualization of the anatomy. Accurate bloodless dissection was possible due to the extremely precise platform, tremor elimination, and significant motion scaling. This was particularly important since the RGE-LNF is small, and the robotic intervention allowed preservation of the lymph nodes and the fine lymphatic channels close to the vascular pedicle. In addition, it also aided in the artery and vein dissection before the final flap pedicle division.

Robot-assisted harvest of the RGE-LNF is feasible and reproducible. It represents an alternative method for flap harvest and paves the way for use of this approach for harvesting other intra-abdominal organs and tissues for reconstructive surgery. This may prove useful for patients who desire the application of the latest technology and less invasive procedures in their treatment, with the possibility of improved outcomes. It offers technical advantages over endoscopic harvesting and provides better cosmesis when compared to the open surgical technique.

Some factors may limit the use of robotic interventions, such as increased cost, a steep learning curve, and increased operating time. However, the distinct advantages of this innovative surgical approach, such as tremor elimination, motion scaling, high-resolution three-dimensional optics, and superior ergonomics, make a compelling case for the expansion of its application in problem-solving specialties, including plastic and reconstructive surgery.

\section{References}

1. Ciudad P, Maruccia M, Socas J, et al. The laparoscopic right gastroepiploic lymph node flap transfer for upper and lower limb lymphedema: technique and outcomes. Microsurgery 2015 Jul 15 [Epub]. http://dx/doi. org/10.1002/micr.22450.

2. Nguyen AT, Suami H. Laparoscopic free omental lymphatic flap for the treatment of lymphedema. Plast Reconstr Surg 2015;136:114-8.

3. Selber JC, Baumann DP, Holsinger FC. Robotic latissimus dorsi muscle harvest: a case series. Plast Reconstr Surg 2012;129:1305-12.

4. Katz RD, Rosson GD, Taylor JA, et al. Robotics in microsurgery: use of a surgical robot to perform a free flap in a pig. Microsurgery 2005;25:566-9.
Supplemental Video S1. The supplemental digital content is an approximately two-minute video of the robotic right gastroepiploic lymph node flap harvest.

Supplemental data can be found at: http://e-aps.org/src/sm/aps-43210-s001.avi

\section{Induced Hypothermia: Implications for Free Flap Survival}

\author{
Michael W Chu ${ }^{1}$, Anita R Kulkarni ${ }^{2}$, Evan Matros $^{3}$ \\ ${ }^{1}$ Division of Plastic and Reconstructive Surgery, Indiana \\ University School of Medicine, Indianapolis, IN; ${ }^{2}$ Plastic Surgery \\ Institute of Washington, Washington, DC; ${ }^{3}$ Division of Plastic \\ and Reconstructive Surgery, Memorial Sloan-Kettering Cancer \\ Center, New York, NY, USA
}

Correspondence: Michael W Chu

Division of Plastic and Reconstructive Surgery, Indiana University School of Medicine, 545 Barnhill Drive Suite \#232, Indianapolis, IN 46202, USA Tel: +1-317-217-3636, Fax: +1-317-217-3636

E-mail:dr.michael.chu@gmail.com

No potential conflict of interest relevant to this article was reported.

Received: 15 Oct $2015 \bullet$ Accepted: 14 Dec 2015

pISSN: 2234-6163 • elSSN: 2234-6171

http://dx.doi.org/10.5999/aps.2016.43.2.212

Arch Plast Surg 2016;43:212-214

Copyright (C) 2016 The Korean Society of Plastic and Reconstructive Surgeons This is an Open Access article distributed under the terms of the Creative Commons Attribution Non-Commercial License (http://creativecommons.org/licenses/by-nc/4.0/) which permits unrestricted non-commercial use, distribution, and reproduction in any medium, provided the original work is properly cited.

Plastic surgeons may not be aware that the new advanced cardiac life support (ACLS) guidelines released in 2010 include induced hypothermia as an adjuvant treatment to improve neurologic outcomes following cardiac arrest [1]. Induced hypothermia (IH) is thought to mitigate ischemic damage by lowering metabolic demand and preventing noxious byproducts of resuscitation. $\mathrm{IH}$ is recommended for patients who experience the return of spontaneous circulation after cardiac arrest, but remain unconscious [2]. The hypothermia protocol involves the reduction of body temperature to $32^{\circ} \mathrm{C}-34^{\circ} \mathrm{C}$ for 12 to 24 hours, followed by slow rewarming $[1,2]$. However, the use of induced hypothermia in microvascular free tissue transfer has not been studied. We present the case of a patient who underwent $\mathrm{IH}$ following free-flap reconstruction of a 